\title{
Temperature Dependence of Macrobending Loss in All-fiber Bend Loss Edge Filter
}

\author{
Pengfei Wang \\ Technological University Dublin, pengfei.wang@tudublin.ie \\ Yuliya Semenova \\ Technological University Dublin, yuliya.semenova@tudublin.ie \\ Gerald Farrell \\ Technological University Dublin, gerald.farrell@tudublin.ie
}

Follow this and additional works at: https://arrow.tudublin.ie/engscheceart

Part of the Engineering Commons

\section{Recommended Citation}

Wang, P., Semenova, Y., Farrell, G. (2008) Temperature dependence of macrobending loss in all-fiber bend loss edge filter. Optics Communications, Vol. 281, pp. 4312-4316, 2008. doi:10.1016/

j.optcom.2008.04.050

This Article is brought to you for free and open access by the School of Electrical and Electronic Engineering at ARROW@TU Dublin. It has been accepted for inclusion in Articles by an authorized administrator of ARROW@TU Dublin. For more information, please contact arrow.admin@tudublin.ie, aisling.coyne@tudublin.ie, gerard.connolly@tudublin.ie.

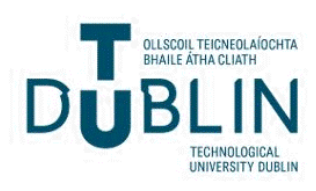




\title{
Temperature dependence of macrobending loss in all-fiber bend loss edge filter
}

\author{
Pengfei Wang, Yuliya Semenova, Gerald Farrell \\ Applied Optoelectronics Center, School of Electronic and Communications Engineering, Dublin \\ Institute of Technology, Kevin Street, Dublin 8, Ireland \\ E-mail: pengfei.wang@ student.dit.ie
}

\begin{abstract}
A theoretical model for macrobending-induced temperature dependent loss (TDL) for a standard singlemode fiber (SMF28) with dual coating layers is presented, with good agreement demonstrated between theoretical calculations and experimental results. The impact of temperature on two examples of an all-fiber based edge filter is also investigated theoretically and experimentally and using the developed model, it is shown that it is possible to predict the impact of temperature variations on an all-fiber based edge filter.
\end{abstract}

Keyword: Temperature dependent loss, macrobending loss, singlemode fiber, SMF28, edge filter

\section{Introduction}

It is well known that temperature change causes significant temperature dependent loss at a bend in a singlemode fiber. Temperature dependence for fiber macrobending loss has been presented in several published papers both theoretically and experimentally [1-4]. The fibers employed in most of the previous published works had a structure that consisted of a fiber core, cladding and a single coating layer.

The influence on bend loss of whispering gallery modes (WGMs) is an important issue considered by many authors, with some publications considering the practical application of WGMs in developing an interferometric sensor [3, 4]. WGMs in bent optical fibers are excited when the radiating rays are reflected at interfaces such as the coating-air boundary or cladding-coating boundary. Such WGMs can recouple to the propagating mode in the fiber core and can result in wavelength dependent 
interference effects.

Most existing commercial standard singlemode fibers (such as SMF28) have two coating layers, which offer protection and stability to the fiber core and cladding. SMF28 fiber has been widely employed for optical communication and sensing. However, previously published investigations of the temperature dependent loss of bending fiber have been focused on the prediction of phase shift in the core modes induced by interference and no existing formulas have been presented for the modeling of theoretical temperature dependent loss of standard singlemode fiber with multiple coating layers. In this paper, the theoretical prediction of temperature dependent loss for an SMF28 fiber based on perturbation theory is presented for the first time.

One justification for the investigation is that recently macrobending singlemode fiber with an absorbing layer (such as SMF28 or 1060XP) has been investigated and optimized for use in a bend loss based edge filter for a rapid wavelength measurement system [5-7]. Changes in macrobending loss with temperature will alter the spectral responses of the fiber edge filter leading to inaccuracy in the measurement of wavelength.

For the bent SMF28 singlemode fiber employed in this paper, the existence of the coating layers produces WGMs for the bent fiber because of reflections at the interfaces at the boundaries of the cladding-coating, the inner and outer coating layers and the coating-air layers. However, for a macrobending fiber based edge filter application, in order to maintain a quasi-linear filter transmission response with wavelength [5], an absorbing layer is required on the surface of the outer coating layer to absorb the radiated light in the outer coating and thus suppress the formation of WGMs at the coating-air interface. In the model the absorption layer is taken account of by assuming an infinite outer coating thickness.

In this paper, we report on both a theoretical and an experimental investigation of the bending induced temperature dependent loss of standard SMF28 singlemode fiber. The operating temperature is controlled within the range $0^{\circ} \mathrm{C}$ to $70^{\circ} \mathrm{C}$, as the SMF28 polymer coatings would suffer degradation if the temperature is higher than $70^{\circ} \mathrm{C}$. The corresponding experimental results show a good agreement with the results of the theoretical modeling. Both the theoretical and experimental results suggest that both the thermal expansion coefficient (TEC) and thermo-optic coefficient (TOC) of the fiber materials have a significant influence on macrobending loss.

\section{Modeling for temperature dependent loss of singlemode fiber with dual}

\section{coating layers}

As is well known, the refractive index of an optical material depends on temperature and wavelength. For silica material, the refractive index varies with the temperature as a result of lattice deformations in the silica matrix structure. For a fiber polymer coating material, the variation of the refractive index is induced by the amplitude of vibrations of polymer molecules and results in the translation and 
distortion of the cross-linked network within the polymer long molecule structure.

A series of theoretical formulations have been developed [8-12] for the prediction of the macrobending loss of singlemode fibers. For example, the theoretical modeling of fiber bending loss based on weak perturbance of the guide field for a multi-coating structure (such as SMF28) has been presented in Ref. [12], along with comparisons with the modeling of fiber macrobending loss which were presented in previous publications [8-11]. Based on the satisfactory agreement between the calculated and experimental results for SMF28 fiber in Ref. [12], the model presented in Ref. [12] is employed in this paper to predict the temperature dependent loss of bending SMF28 singlemode fiber.

Fig. 1 gives the schematic cross-section of a bending fiber with multiple cladding or coating layers. The bending radius is denoted by $R$. For the $q$-th $(q$ is a positive integer, $q=1,2,3 \ldots)$ layer of fiber, its refractive index is $n_{q}$ and the thickness is $x_{q}-x_{q-1}$. $q=1$ represents the core and $q=2$ represents the cladding layer.

As a starting point, the analysis in Ref. [10] is used, In Ref. [10], the fiber was assumed to consist of a core, cladding $(q=2)$ and an infinite coating $(q=3)$. When the fiber is bent, the Fourier transform scalar field in the cladding and infinite coating regions $(q=2,3)$ in the $y$-direction can be expressed as:

$$
\nabla_{t}^{2} \bar{\psi}_{q}(x, \zeta)+\left[k^{2} n_{q}^{2}\left(1+\frac{2 x}{R}\right)-\beta_{0}^{2}-\zeta^{2}\right] \bar{\psi}_{q}(x, \zeta)=0
$$

where $\bar{\psi}_{q}(x, \zeta)$ is the Fourier transform along the $y$-direction of the transverse field component in region $q$; $\zeta$ is the conjugate variable for the Fourier transform in $y$-direction; $n_{q}$ is the refractive index in region $q ; k$ is the well know wavenumber in vacuum at the wavelength $\lambda$, it can be expressed as $k=2 \pi / \lambda ;\left(1+\frac{2 x}{R}\right)$ is the correction factor for $n_{q}^{2}$ to take account of the change induced by the stress-optic effect; and $\beta_{0}$ is the complex propagation constant, the imaginary part of which is the bend loss coefficient. Following solution by an inverse Fourier transform of the y-field, equation (1) can be treated as [11]:

$$
\psi_{q}(x, y)=\frac{1}{2 \pi} \int_{-\infty}^{+\infty}\left[D_{q}(\zeta) B_{i}\left(X_{q}\right)+H_{q}(\zeta) A_{i}\left(X_{q}\right)\right] \cdot \exp (-i \zeta y) d \zeta
$$

where $X(x, \zeta)=\left(\frac{R}{2 k^{2} n_{q}^{2}}\right)^{2 / 3}\left[\beta^{2}+\zeta^{2}-k^{2} n_{q}^{2}\left(1+\frac{2 x}{R}\right)\right]$, and $B_{i}$ and $A_{i}$ are Airy functions, respectively. Both $D_{q}(\zeta)$ and $H_{q}(\zeta)$ are the Fourier spectra which were 
previously defined in Ref. [10]. For the outermost infinite layer, the relation between $D_{q}(\zeta)$ and $H_{q}(\zeta)$ can be defined as $H_{q}(\zeta)=-i D_{q}(\zeta)$. Thus by extending the model, for any two adjacent layers in the fiber structure, given the continuous boundary conditions of the field, the adjacent fields are given by [12]:

$$
\left\{\begin{array}{l}
D_{q}(\zeta) B_{i}\left[X_{q}\left(x_{q}, \zeta\right)\right]+H_{q} A_{i}\left[X_{q}\left(x_{q}, \zeta\right)\right]=D_{q+1}(\zeta) B_{i}\left[X_{q}\left(x_{q+1}, \zeta\right)\right]+H_{q+1} A_{i}\left[X_{q}\left(x_{q+1}, \zeta\right)\right] \\
D_{q}(\zeta) B_{i}^{\prime}\left[X_{q}\left(x_{q}, \zeta\right)\right]+H_{q} A_{i}^{\prime}\left[X_{q}\left(x_{q}, \zeta\right)\right]=D_{q+1}(\zeta) B_{i}^{\prime}\left[X_{q}\left(x_{q+1}, \zeta\right)\right]+H_{q+1} A_{i}^{\prime}\left[X_{q}\left(x_{q+1}, \zeta\right)\right]
\end{array}\right.
$$

which has the form of $\left[\begin{array}{l}D_{j}(\zeta) \\ H_{j}(\zeta)\end{array}\right]=\left[\begin{array}{ll}M_{11} & M_{12} \\ M_{21} & M_{22}\end{array}\right]\left[\begin{array}{l}D_{j+1}(\zeta) \\ H_{j+1}(\zeta)\end{array}\right]$. Considering the core and cladding layer, the relationship between $D_{1}(\zeta)$ and $H_{1}(\zeta)$ is obtained as the following

$$
D_{1}(\zeta)=\frac{M_{11} i+M_{12}}{M_{21} i+M_{22}} H_{1}(\zeta)=G H_{1}(\zeta)
$$

Given the boundary condition at the interface between the core layer and cladding layer, then:

$$
D_{1}(\zeta) B_{i}\left[X_{2}\left(x_{1}, \zeta\right)\right]+H_{1}(\zeta) A_{i}\left[X_{2}\left(x_{1}, \zeta\right)\right]=\frac{\pi}{\sqrt{\gamma^{2}+\zeta^{2}}} \exp \left(-a \sqrt{\gamma^{2}+\zeta^{2}}\right)
$$

Therefore, $H_{1}(\zeta)=\frac{\frac{\pi}{\sqrt{\gamma^{2}+\zeta^{2}}} \exp \left(-a \sqrt{\gamma^{2}+\zeta^{2}}\right)}{G B_{i}\left[X_{1}\left(x_{1}, \zeta\right)\right]+A_{i}\left[X_{1}\left(x_{1}, \zeta\right)\right]}$ and finally acco
perturbation theory the bend loss factor can be expressed as:
\[ 2 \alpha=-2 \frac{\kappa^{2}}{2 \pi \beta V^{2} K_{1}^{2}(a \gamma)} \operatorname{Im}\left(\int_{-\infty}^{\infty} H_{1}(\zeta) A_{i}\left[X_{2}(0, \zeta)\right] d \zeta\right) \]

This factor can be used for the calculation of bend loss for a fiber with one or more coating layers, and the above formulas are used in the investigation which follows of the temperature dependence of bending loss for SMF28 fiber.

In most previously published papers [9-11], the effective bend radius (a wavelength dependent correction factor) was employed to take account of stress induced refractive index changes in order to achieve a good agreement between the modeled results and the experimental results. However in our previously published work [12], the good agreement between the theoretical model and experimental results for Corning standard singlemode fiber-SMF28 showed that for this particular fiber, the so-called "effective bending radius" used to take account of macrobending stress, was not required. SMF28 is the fiber type employed in the investigations of 
temperature dependent loss in this paper. For the theoretical model employed in this paper, to maintain generality, the stress-optic effect is included in the model and all formulas, but in applying the model for SMF28 fiber, the value of the correction factor is set equal to 1 .

For the fiber used in this paper the key parameters at $20^{\circ} \mathrm{C}$ are shown in Table 1:

While most of the parameter values are published by the manufacturer of SMF28, some TEC and TOC values were extracted from Ref. [13].

With the parameters of TEC and TOC listed in the Table 1, the corresponding temperature dependent thickness and refractive index of each fiber layer at a temperature $T$ can be expressed as:

$$
\begin{gathered}
x_{T}=\left(x_{q}-x_{q-1}\right) \cdot \alpha_{q} \cdot \Delta T \\
n_{T}=n_{q} \cdot \beta_{q} \cdot \Delta T
\end{gathered}
$$

where $x_{q}$ is the distance from the fiber core center to the $q$-th fiber layer boundary as shown in Figure 1; $\alpha_{q}$ is the TEC of the $q$-th layer of fiber material, $\beta_{q}$ is the TOC of the $q$-th layer of fiber material and $\Delta T$ is the difference in temperature with respect to $20^{\circ} \mathrm{C}$.

A bending singlemode SMF28 fiber with dual coating layers and an absorbing layer can be treated as core-cladding-primary coating-infinite coating structure. In the previous publications [8-12], it was found that radiation, which is launched from the fiber core at the bend enters the coating region(s) of the fiber and thus it is important in principle to consider the effect of absorption within the coating in modeling the behavior of a bent fiber. However in the theoretical model in this case, we assume an infinite outer coating thickness, which suppresses the evolution of strong WGMs that otherwise could arise at the coating-air interface, where the refractive index difference is 0.5294. Experimentally an absorbing layer is applied to the surface of the outer fiber coating to achieve the same effect. WGMs also evolve at the interface between the coatings, where there is a small refractive index change (0.0508). However the actual absorption coefficient of the coating layers is in any event very low. Given that the coating layers are 62.5 microns thick, the coating absorption loss is less than 0.02 $\mathrm{dB}$ at a wavelength of $1550 \mathrm{~nm}$ and is thus not considered significant enough for inclusion in the model.

In Figure $2(a)$, the calculated bend losses are shown for two different temperatures $0^{\circ} \mathrm{C}$ and $70^{\circ} \mathrm{C}$. From this figure one can see that the bend loss at either temperature does not increase monotonically as the bending radius decreases. For example, bend losses for a bending radius of $9 \mathrm{~mm}$ are larger than those for a bending radius of $8.5 \mathrm{~mm}$. This is due to the coherent coupling between the fundamental propagation mode and the reflected radiation from two interfaces: the cladding-inner coating interface and the inner coating-outer coating interface. These so-called whispering-gallery modes have a significant effect on the bend loss characteristics. 
The temperature dependent parameters TOC and TEC have a significant impact on the interference process and thus there are a significant changes in bend loss with temperature, which are also sensitive to the bend radius value, in particular below 9.5 $\mathrm{mm}$. Overall a consequence of the fact that bend loss is not a monotonic function of bend radius is that when the temperature changes from $0^{\circ} \mathrm{C}$ and $70^{\circ} \mathrm{C}$, the TDL at different bent radii (shown in Figure 2 (b)) displays significant variations in value and also changes sign.

Figure $2(b)$ shows the calculated difference in macrobending loss for two temperatures, $0^{\circ} \mathrm{C}$ and $70^{\circ} \mathrm{C}$, as a function of different bend radius ranging from 8 to $12.5 \mathrm{~mm}$, where the bending fiber length is 10 turns and the wavelength is $1550 \mathrm{~nm}$. The calculation was carried out by applying the corresponding TEC and TOC parameters of SMF28 fiber into the theoretical model described above. In Fig. $2(b)$, one can also see that the calculated macrobending loss difference between $0^{\circ} \mathrm{C}$ and $70^{\circ} \mathrm{C}$ is very sensitive to the bend radius when the bend radius ranges from 8 to 9.5 $\mathrm{mm}$ due to the significant difference of bend losses between $0^{\circ} \mathrm{C}$ and $70^{\circ} \mathrm{C}$ presented in Fig. $2(a)$. The maximal bend loss difference in Fig. $2(b)$ is $-32.67 \mathrm{~dB}$ for a bend radius of $9 \mathrm{~mm}$.

\section{Experimental results and discussion}

Fig. 3 shows the experimental setup used for the measurement of temperature dependent loss; the SMF28 singlemode fiber is wrapped to form bend loops around a series of precisely dimensioned metal mandrels, with each mandrel providing a different usable diameter. The beginning and end of the bending fiber is fixed by a two-part epoxy to improve mechanical stability. The bending fiber was connected to a tunable laser and an optical spectrum analyser. The tunable laser has an output power range from $+7 \mathrm{dBm}$ to $-7 \mathrm{dBm}$. Both the bending fiber with an outer absorbing layer and mandrel were attached to a thermoelectric Peltier cooler, which is controlled by a digital Temperature Controller (ITC 510, Thorlabs), while a digital resistance thermometer sensor probe is attached to the mandrel to accurately measure the temperature.

As presented in Ref. [1-3, 14], a bending singlemode fiber without an outer absorbing layer is attractive for applications such as thermo-optic interferometric sensing using the so-called whispering gallery mode, as fiber bends have a significant influence on the whispering gallery mode. However, a bending SMF28 fiber which does have an absorbing layer is needed in an all-fiber ratiometric wavelength measurement application $[6,12]$. Thus the temperature dependent measurements of bending fiber are carried out with an absorbing layer to suppress the whispering gallery mode.

In order to better characterize the temperature dependent loss, the temperature dependent loss value is defined as the difference in bend loss between $0^{\circ} \mathrm{C}$ and $70^{\circ} \mathrm{C}$ thus:

$$
T D L=B L_{70^{\circ} \mathrm{C}}-B L_{0^{\circ} \mathrm{C}}
$$


Using this formula, the measurements of bend loss of the SMF28 are carried out for discrete bending radii ranging from 8 to $12 \mathrm{~mm}$ at a wavelength of $1550 \mathrm{~nm}$ at temperatures of $0^{\circ} \mathrm{C}$ and $70^{\circ} \mathrm{C}$. In practice, because of the finite diameter of SMF28 fiber, the radius of the fiber should be treated as a part of bend radius, thus $0.125 \mathrm{~mm}$, the radius of SMF28 fiber, is added to each mandrel radius. In Fig. 4, both the theoretical modeling (solid line) and experimental temperature dependent loss results (star points) as a function of different bending radii are presented, from which one can see that the theoretical modeling of temperature dependent loss shows a reasonable agreement with the experimental results. The discrepancy between the calculated temperature dependent loss and measured results could be induced by the limited physical accuracy of each bend radius and the scalar approximation modeling of bending loss.

The primary concern in this work is to study the impact of temperature on an all-fiber based edge filter. Using the model above, it is possible to predict the impact of temperature variations on an all-fiber based edge filter. We investigate this temperature dependence by measuring the bend loss for two different edge filters over the range $0^{\circ} \mathrm{C}$ and $70^{\circ} \mathrm{C}$, in $5^{\circ} \mathrm{C}$ increments. Both of the edge filters have bending radii equal to those which have been investigated in our previous publication [15]. The two cases of fiber edge filters are, case 1) a bending length of 10 turns (is 667.59 $\mathrm{mm}$ ) for the bending radius of $10.5+0.125 \mathrm{~mm}$ and case 2) a bending length of 20 turns (is $1272.35 \mathrm{~mm}$ ) for a bending radius of $10+0.125 \mathrm{~mm}$,

In the measured bend loss results, both the transition loss which exists in the section between the bent section and the straight fiber, and the insertion loss which includes the interconnection loss and splicing loss, are deducted from the total transmission loss to obtain the "pure" bend loss within the bent section.

In Figure $5(a)$ and $5(b)$, the variation in the edge filter loss with temperature at $1550 \mathrm{~nm}$ is presented for two different bend radii/bend length combinations. The Figures show both experimental and modeled results for both single and dual coating layer models. The calculated TDLs using a dual coating layer model are in reasonable overall agreement with the experimental results. It is clear also that the single layer model, while it may be accurate at predicting loss at a fixed temperature of $20^{\circ} \mathrm{C}$, is not adequate for predicting the variation of loss with temperature. Also from Fig. 5 (a) and $(b)$, one can see that wave-like variations exist in both modeled and measured temperature dependent loss results.

A physical interpretation of these results is as follows. In the theoretical model with single-coating layer, the fiber coating is assumed to be infinitely thick and hence the radiation entering the coating layer from the cladding propagates away from the fiber without any subsequent reflection. This explanation is consistent with the monotonic behaviour of the dashed-dot curves in Fig.5 $(a)$ and $(b)$. In the theoretical model with dual-coating layer, the primary coating layer has a finite thickness and the secondary coating layer is assumed to be infinitely thick. Hence radiation entering the primary coating from the cladding is partially reflected at the first interface of the cladding-primary coating layer, and again at the second interface of the primary-secondary coating layer. The finite thickness of the primary coating layer 
combined with the index differences on either side form in effect a simple Fabry-Perot interferometer. Therefore as both the refractive index and thickness of the primary coating varies with changes in temperature, at a fixed wavelength, the fraction of incident radiation in the primary coating that is reflected back into the cladding toward the core will vary periodically. This would account for the oscillations in the solid curves in the Fig.5 (a) and $(b)$.

In Figure $5(a)$ and $5(b)$, the discrepancy between the calculated TDLs and measured results could be caused by: 1) the approximations made in the calculation. The scalar approximation theory is built on a base of perturbation theory, and the interface of between the fiber cladding and infinite coating layer was treated as an infinite plane and the light field within the core is approximated by the unperturbed field of the straight fiber with an infinite cladding as mentioned in Section 2; 2) Temperature induced mechanical stress, for example asymmetric expansion between the primary and secondary coating layer as temperature changes. In table 1, one can see that the TEC values for the primary and secondary coatings are significantly different. This means that as temperature changes, varying stress forces will be induced between the coatings, increasing overall stress and perturbing the loss measurements. The theoretical and experimental work is ongoing on these issues.

\section{Conclusion}

In this paper, macrobending induced temperature dependent losses for a standard singlemode fiber (SMF28) have been investigated both theoretically and experimentally. The calculations of temperature dependent loss of bending SMF28 fiber are also presented based on perturbation theory and it shown that the developed model can be utilized to successfully predict the temperature dependence of an all-fiber bend loss edge filter, where the fiber has a dual coating.

\section{Reference}

1. R. Morgan, J. S. Barton, P. G. Harper and J. D. C. Jones, "Temperature dependence of bending loss in monomode optical fibres," Electron. Lett. Vol. 26 No. 13, pp. 937-939 (1990).

2. F. M. Haran, J. S. Barton and J. D. C. Jones, "Bend loss in buffered over-moded optical fibre: $\mathrm{LP}_{11}$ mode and 'whispering gallery' mode interaction," Electron. Lett. Vol. 30 No. 17, pp. 1433-1434 (1994).

3. F. M. Haran, J. S. Barton, S. R. Kidd and J. D. C. Jones, "Optical fibre interferometric sensors using buffer guided light,” Meas. Sci. Technol. Vol. 5, pp. 526-530 (1994).

4. Y.-G. Han, J. H. Lee and S. B. Lee, "Discrimination of bending and temperature sensitivities with phase-shifted long-period fiber gratings depending on initial coupling strength," Opt. Exp. Vol. 12 No. 14, pp. 3204-3208 (2004).

5. Q. Wang, G. Farrell, T. Freir, G. Rajan, and P. Wang, "Low-cost wavelength measurement based on a macrobending single-mode fiber," Opt. Lett., Vol. 31, No. 12, pp. 1785-1787 (2006). 
6. Q. Wang, G. Farrell and T. Freir, "Study of transmission response of edge filters employed in wavelength measurements," Appl. Opt., Vol. 44, No.36, pp. 7789-7792 (2005).

7. P. Wang, G. Farrell, Q. Wang and G. Rajan, "An optimized macrobending-fiber-based edge filter”, IEEE Photon. Technol. Lett., Vol. 19, No. 15, pp. 1136-1138 (2007).

8. C. Vassallo, "Perturbation of a LP mode of an optical fibre by a quasi-degenerate field: a simple formula," Opt. \& Quantum Electron. Vol. 17, No. 3, pp. 201-205 (1985).

9. I. Valiente and C. Vassallo, "New formalism for bending losses in coated single-mode optical fibres," Electron. Lett., Vol. 25, No. 22, pp. 1544-1545 (1989).

10. H. Renner, "Bending losses of coated single-mode fibers: a simple approach," J. Lightwave Technol., Vol. 10, No. 5, pp. 544-551 (1992).

11. L. Faustini and G. Martini, "Bend loss in single-mode fibers," J. Lightwave Technol., Vol. 15, No.4, pp. 671-679 (1997).

12. Q. Wang, G. Farrell, and T. Freir, "Theoretical and experimental investigations of macro-bend losses for standard single mode fibers," Opt. Exp., Vol. 13, No. 12, pp. 4476-4484 (2005).

13. C.-C. Lai, W.-Y. Lee and W.-S. Wang, "Gamma radiation effect on the fiber Fabry-Pérot interference sensor," IEEE Photon. Technol. Lett., Vol. 15, No. 8, 1132-1134 (2003).

14. S. H. Nam and S. Yin, "High-temperature sensing using whispering gallery mode resonance in bent optical fibers," IEEE Photon. Technol. Lett., Vol. 17, No. 11, pp. 2391-2393 (2005).

15. Q. Wang, G. Rajan, P. Wang, and G. Farrell, "Polarization dependence of bend loss for a standard singlemode fiber," Opt. Express, Vol. 15, No. 8, pp. 4909-4920 (2007). 


\section{Figure captions}

Fig. 1 Cross-section of a bending fiber with multiple coating layers.

Fig. 2 (a) Calculated macrobending loss for SMF28 fiber at the temperature of $0^{\circ} \mathrm{C}$ and $70^{\circ} \mathrm{C}$, respectively; (b) Calculated result of difference of macrobending loss for SMF28 fiber between $0^{\circ} \mathrm{C}$ and $70^{\circ} \mathrm{C}$ at the wavelength of $1550 \mathrm{~nm}$, the bending length is 10 turns.

Fig. 3 Experimental setup for measuring temperature dependent loss of macrobending fiber.

Fig. 4 Calculated and measured bend loss of SMF28 as a function of bend radius at wavelength $1550 \mathrm{~nm}$ with a bending length of 10 turns.

Fig. 5 Modeled and measured macrobending loss results for temperature ranging from 0 to $70^{\circ} \mathrm{C}$ at a wavelength of $1550 \mathrm{~nm}$, the bending radius is a) $10.5+0.125 \mathrm{~mm}$ with a bending length of 10 turns; b) $10+0.125 \mathrm{~mm}$ with a bending length of 20 turns. 


\section{Table caption}

Table 1 Parameters of the standard Corning SMF28 CPC6 dual coating singlemode fiber; (the refractive index values are defined at a wavelength of $1550 \mathrm{~nm}$ at the room temperature, about $20^{\circ} \mathrm{C}$ ) 
Fig. 1

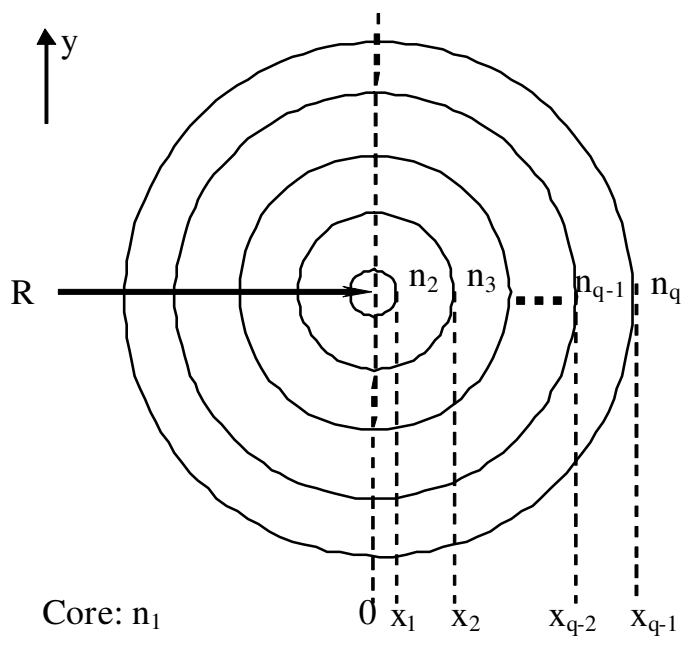


Fig. 2 (a)

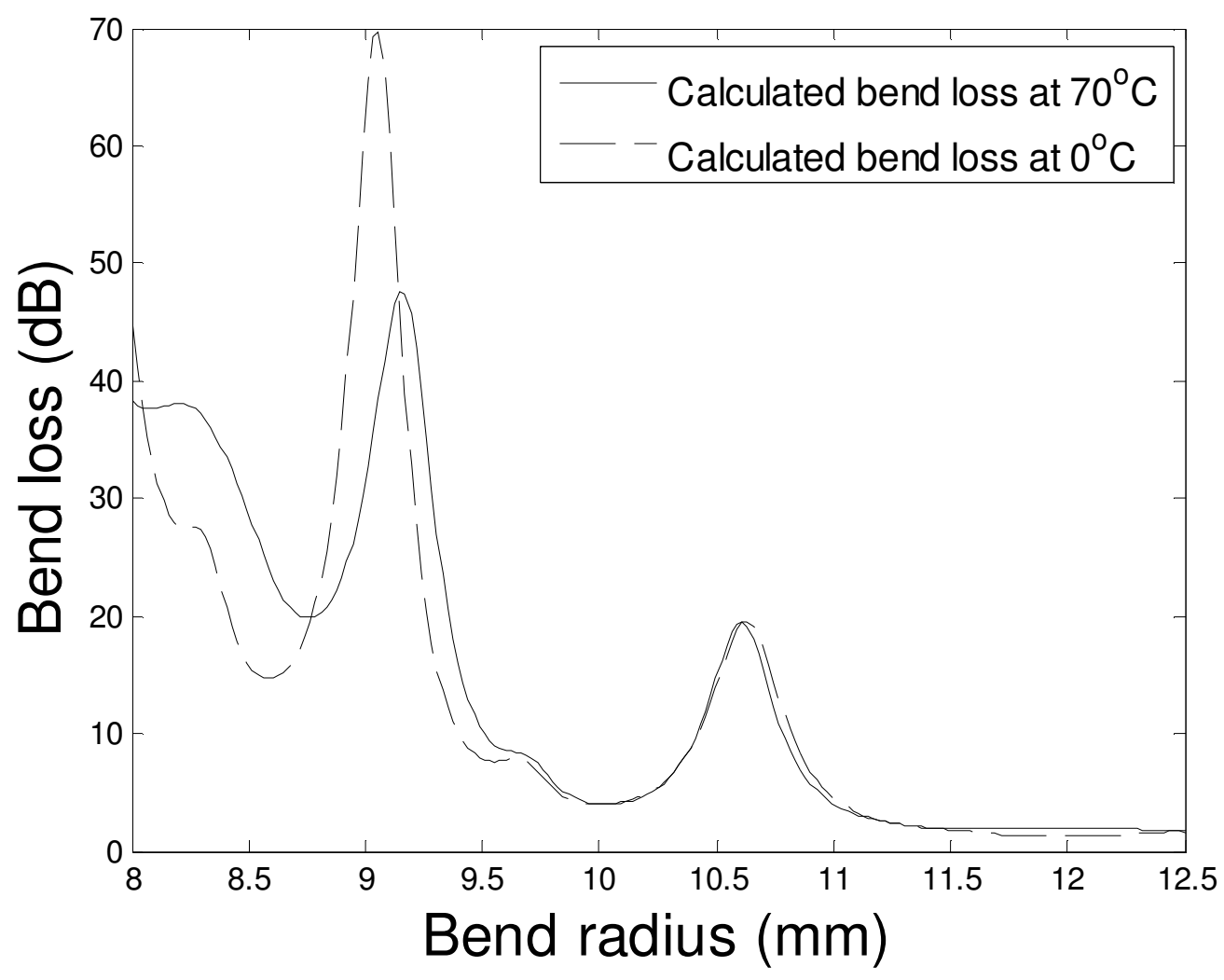


Fig. 2 (b)

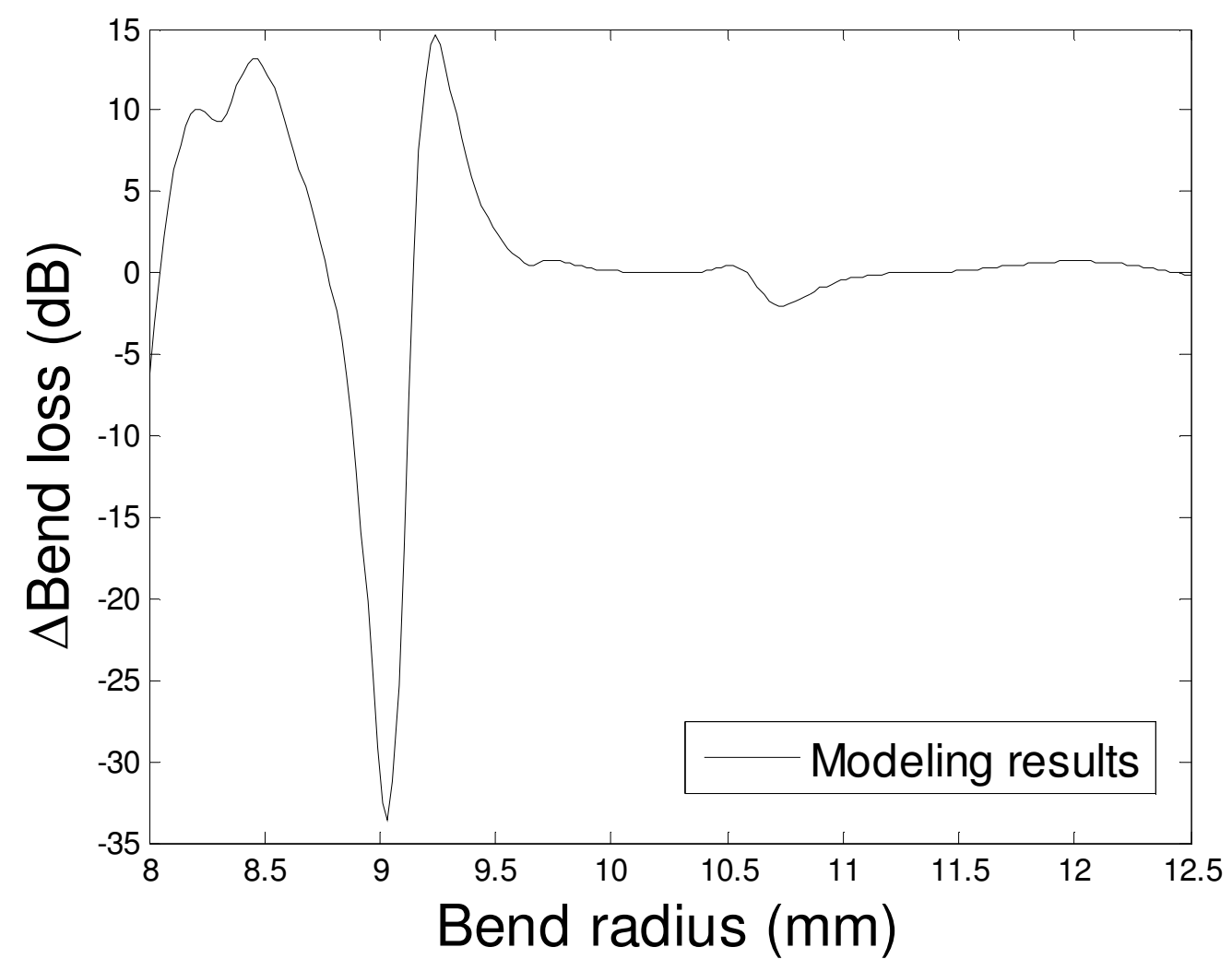


Fig. 3

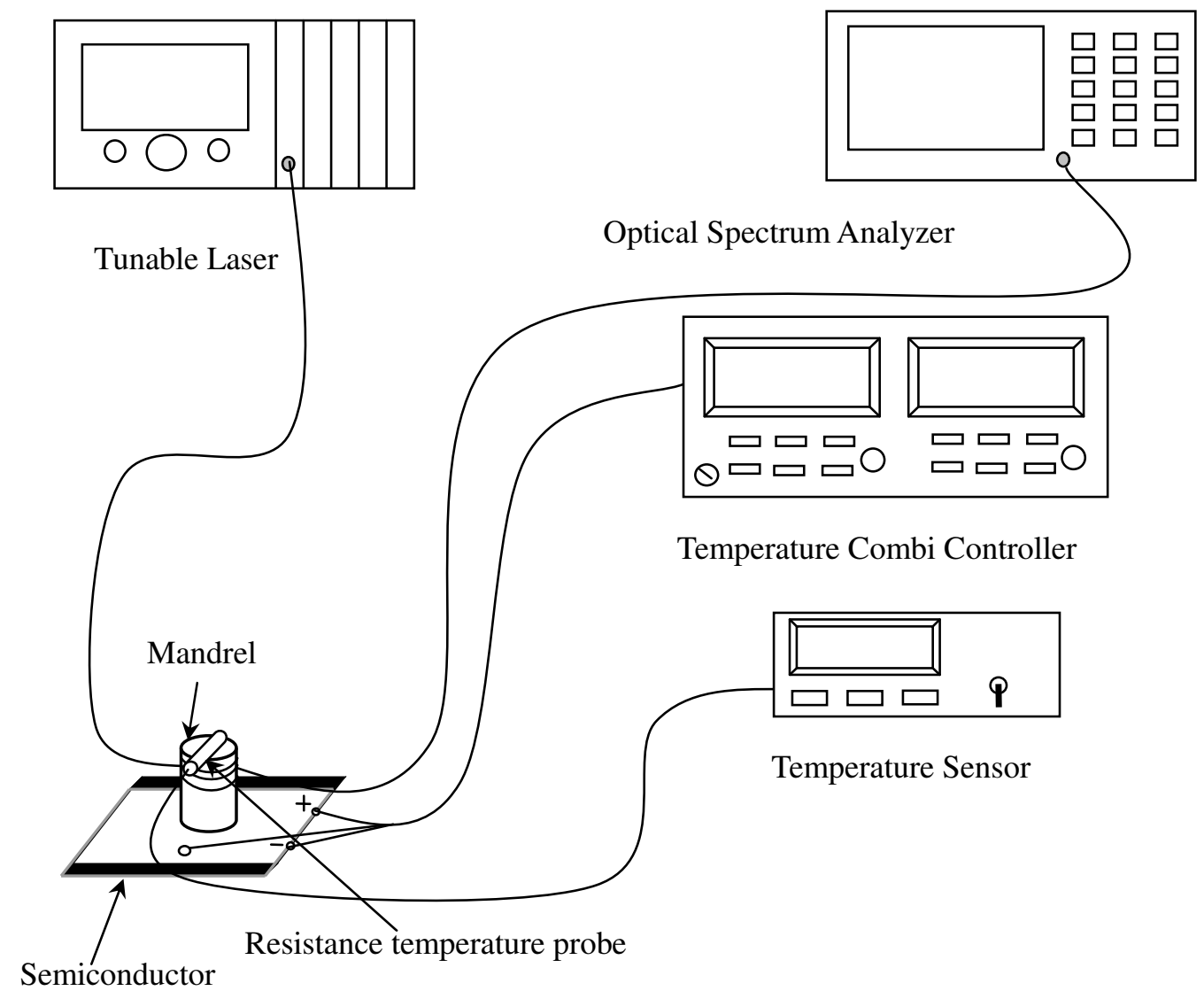

thermo-electric Peltier cooler 
Fig. 4

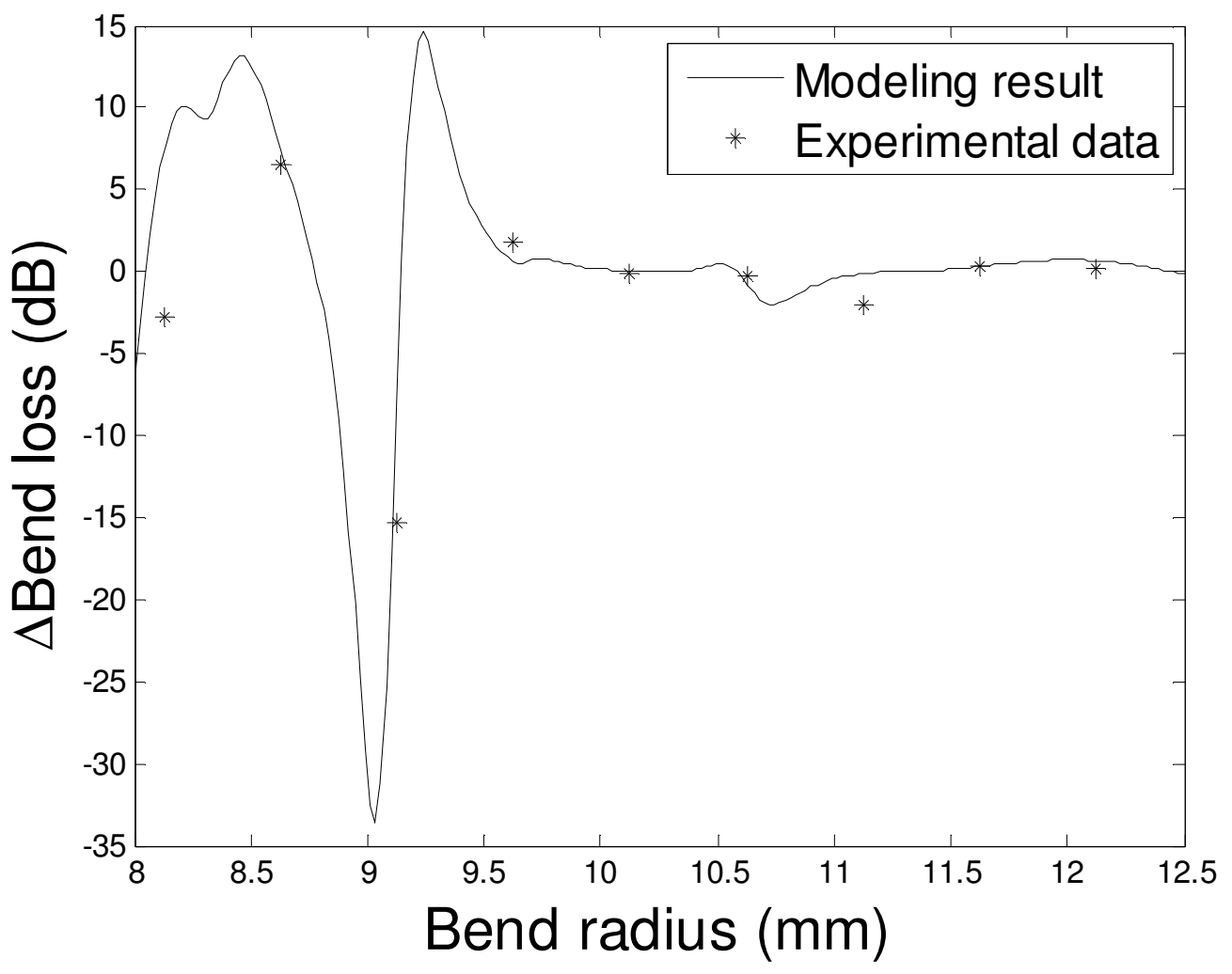


Fig. 5a

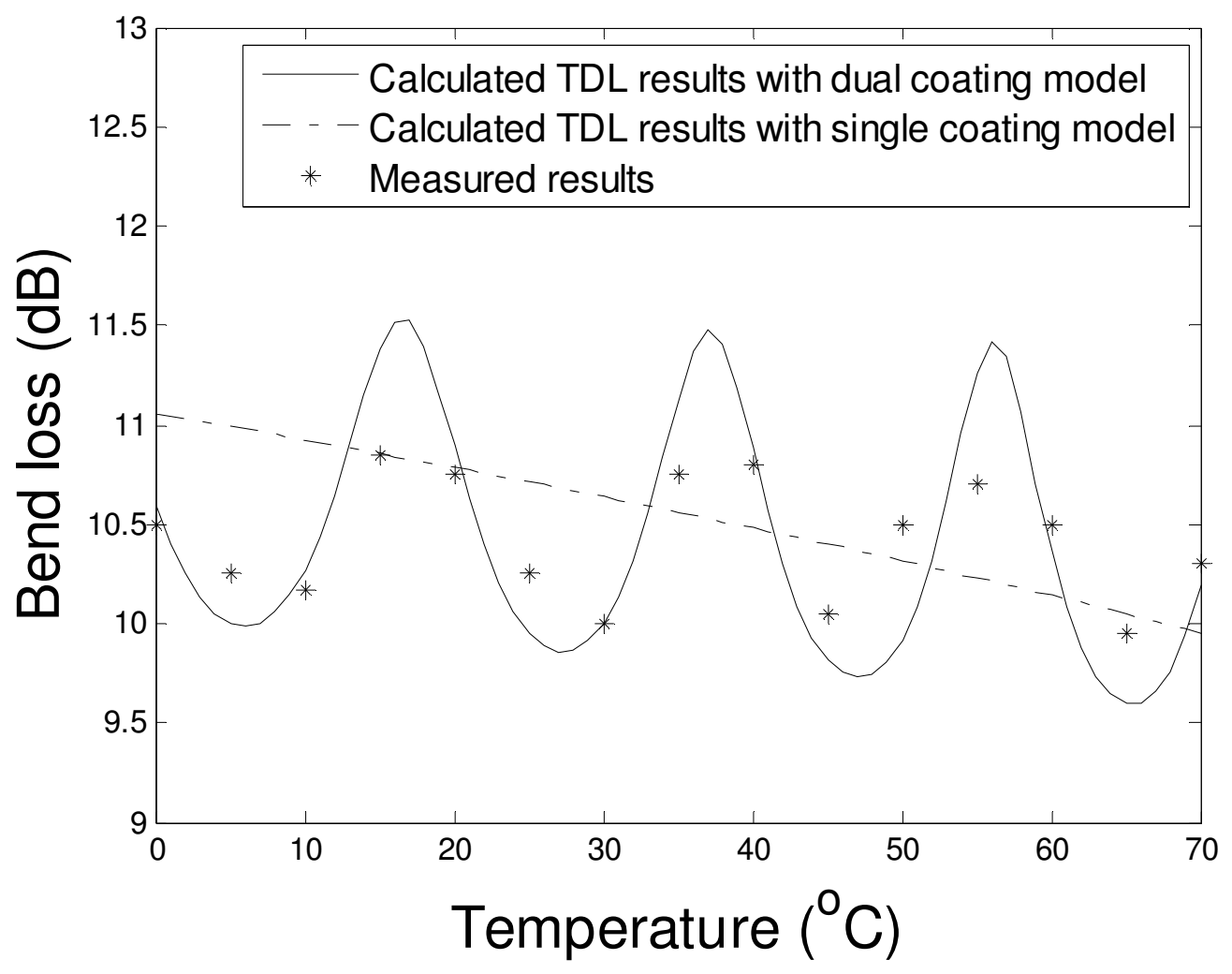


Fig. $5 b$

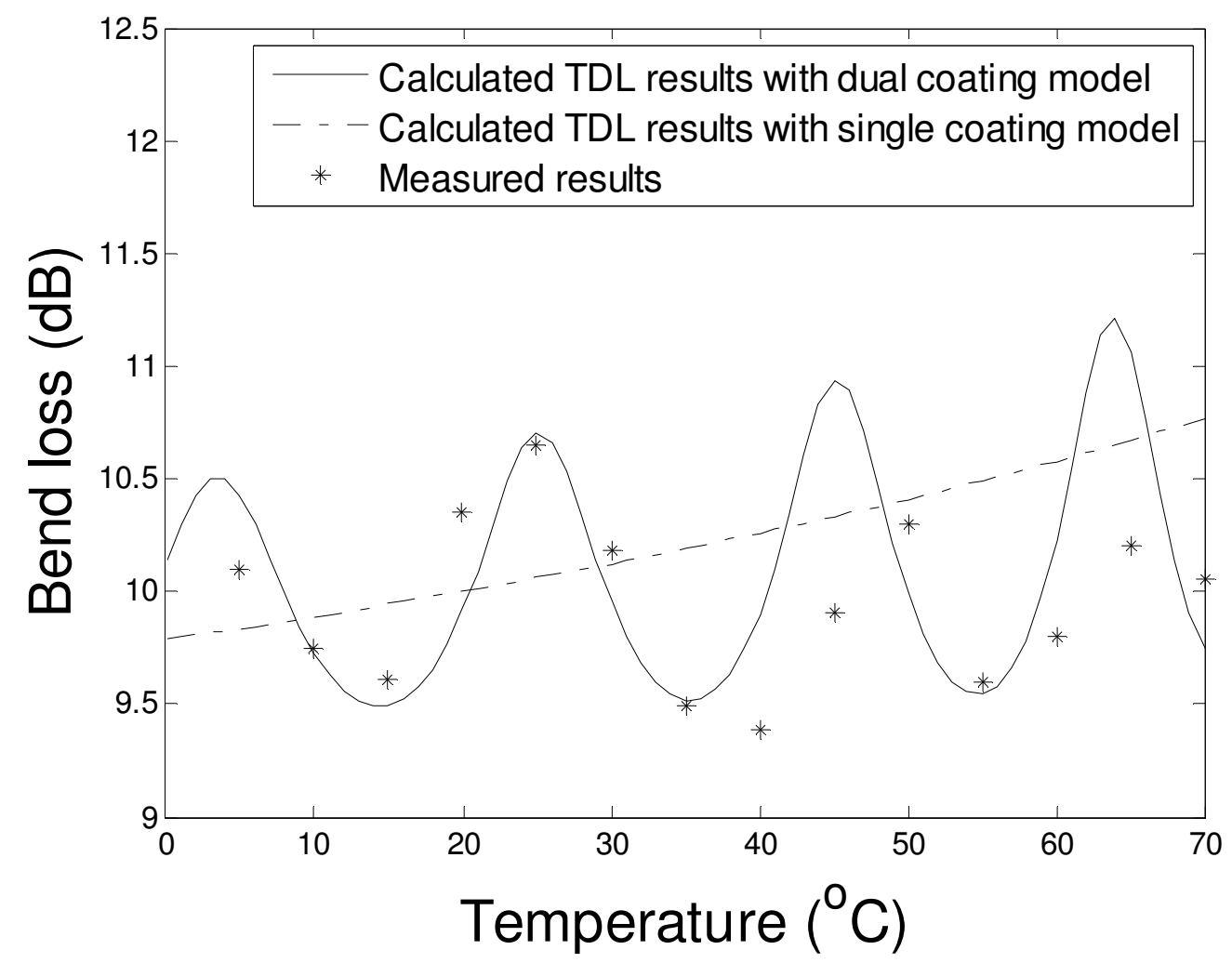


Table 1

\begin{tabular}{ccccc}
\hline \hline SMF28 fiber & Core & Cladding & Primary coating & Secondary coating \\
\hline Refractive index & 1.4504 & 1.4447 & 1.4786 & 1.5294 \\
Radius $(\mu \mathrm{m})$ & 4.15 & 62.5 & 95 & 125 \\
TEC $\alpha\left(\mathrm{K}^{-1}\right)$ & $5.5 \times 10^{-7}$ & $5.5 \times 10^{-7}$ & $800 \times 10^{-7}$ & $<100 \times 10^{-7}$ \\
TOC $\beta\left(\mathrm{K}^{-1}\right)$ & $\approx 1 \times 10^{-5}$ & $\approx 1 \times 10^{-5}$ & $-2.9 \times 10^{-4}$ & - \\
\hline \hline
\end{tabular}

